\title{
Analytical Solutions for Steep Standing Waves
}

\author{
Rodney J. Sobey \\ Department of Civil and Environmental Engineering, \\ Imperial College London, \\ London SW7 2AZ, UK \\ Phone: +44 (0)20 7594 5995, Fax: +44 (0)20 75945991 \\ E-mail: r.j.sobey@imperial.ac.uk
}

\section{Engineering and Computational Mechanics, 162:185-197, 2009. [Errata 16}

\author{
16 November 2011
}

\begin{abstract}
A Stokes-style analytical theory for standing waves is developed and explored. The theory is numerically confirmed as correct to fifth order, and details of the solution are archived in a manner that anticipates the confirmation demands of application code. The uniquely non-linear aspects of the predicted kinematics are demonstrated in parallel with the equivalent linear theory prediction. Finally, the limits of validity of the analytical solution are examined, and lead to a preliminary but pragmatic estimate of limit standing waves.
\end{abstract}

Keywords Analytical theory, extreme wave kinematics, limit waves, short waves, standing waves, unsteady flow.

\section{Introduction}

Steady wave conditions may be a mix of progressive and standing wave modes. Notwithstanding the mode, engineering interest is concentrated on the more extreme wave conditions and on the prediction of the kinematics under such conditions.

The existing predictive capability for the entire spectrum of progressive waves, from small to limit waves and from very shallow depths to very deep water, is now mature and very well established.

The situation for standing waves is much less satisfactory. It is the more analytically (and numerically) demanding mode, as it cannot be reduced to a steady problem in a single horizontal $x$ variable. For a standing wave, both $x$ and $t$ must be retained. Not 


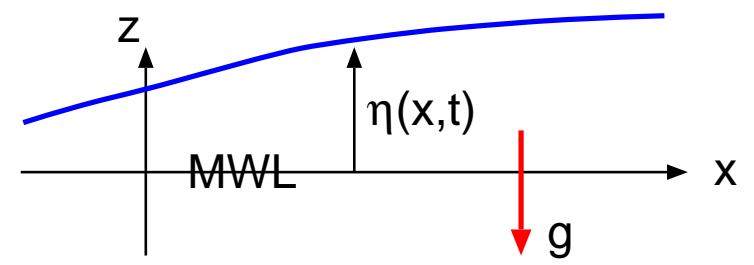

$\mathrm{z}=-\mathrm{h}$

Figure 1: Definition Sketch.

surprisingly perhaps, the literature is much less satisfactory. Analytical theories are inconsistent, presumably a consequence of typographical errors in presenting the often long and complicated coefficient equations at higher orders. Discussions of numerical theories are brief and of uncertain promise, principally as verification against analytical theories remains uncertain.

The present study initially clarifies the uncertain predictive capabilities of existing analytical theories for standing waves. Subsequently, a new Stokes-style analytical theory to fifth order is presented. The fidelity of this new theory is confirmed numerically by Richardson extrapolation to the limit. The response patterns of the nonlinear kinematics are then reviewed, followed by an investigation of the limits of validity of the theory and a preliminary prediction for the limit wave height.

\section{Problem Formulation}

Standing waves in horizontal dimension $x$, vertical dimension $z$ and time $t$ assume irrotational flow such that the field equation is the Laplace equation

$$
\frac{\partial^{2} \phi}{\partial x^{2}}+\frac{\partial^{2} \phi}{\partial z^{2}}=0
$$

in which $\phi(x, z, t)$ is the velocity potential function. $x$ is measured in the plane of the Mean Water level (MWL) with $z$ directed upwards in opposition to the gravitational vector $\vec{g}$ (see Figure 1) The theoretical problem formulation is completed by

(i) the bottom boundary condition $(\mathrm{BBC})$, requiring no flow through the horizontal bed,

$$
w(x, z=-h, t)=0
$$

in which $w=\partial \phi / \partial z$ is the vertical velocity, 
(ii) the kinematic free surface boundary condition (KFSBC), requiring no flow through the instantaneous free surface,

$$
\frac{\partial \eta}{\partial t}+u \frac{\partial \eta}{\partial x}=w \quad \text { at } z=\eta(x, t)
$$

in which $u=\partial \phi / \partial x$ is the horizontal velocity,

(iii) the dynamic free surface boundary condition (DFSBC), requiring atmospheric pressure at the instantaneous free surface,

$$
\frac{\partial \phi}{\partial t}+\frac{1}{2}\left(u^{2}+w^{2}\right)+g \eta=\bar{B} \quad \text { at } z=\eta(x, t)
$$

in which $\bar{B}$ is the Bernoulli constant, and

(iv) the periodic lateral boundary conditions (PLBC), requiring periodicity in both $x$ and $t$

$$
\phi(x, z, t)=\phi(x+2 \pi / k, z, t)=\phi(x, z, t+2 \pi / \omega)
$$

in which $k$ is the wave number and $\omega$ is the wave frequency.

The non-linearity of the standing wave problem is manifest through the free surface boundary conditions, Equations 3 and 4.

\section{Existing Analytical Theories}

Analytical predictions available in the literature are Stokes-style approaches representing both $\eta$ and $\phi$ as small parameter perturbation expansions in a small non-dimensional parameter related to the wave height. Predictions include Penney and Price (1952) to fifth order in deep water and, in transitional water, Tadjbakhsh and Keller (1960) to third order, Goda and Kakizaki (1966) (see also Goda (1967)) to fourth order and Hsu et al. (1979) to third order.

As the dominant engineering interest is prediction in transitional $\left(0.3 \lesssim \omega^{2} h / g \lesssim 2.5\right)$ and shallow water $\left(\omega^{2} h / g \lesssim 0.3\right)$, initial attention was directed to the transitional depth theories. It was immediately apparent that agreement among these theories was relatively poor. The difficulties are presumably a consequence of typographical errors, but much beyond first order, the problems are difficult to identify without recreating the theories.

Confirmation of a verifiably correct analytical theory follows from Richardson extrapolation to the limit, following Fenton (1985a). The procedure is based on the kinematic (Equation 3) and dynamic (Equation 4) free surface boundary conditions, reformulated respectively as

$$
\begin{aligned}
f_{K}(x, t) & =\left.\frac{\partial \eta}{\partial t}\right|_{x, t}+\left.\left.u\right|_{x, \eta(x, t), t} \frac{\partial \eta}{\partial x}\right|_{x, t}-\left.w\right|_{x, \eta(x, t), t} \\
f_{D}(x, t) & =\left.\frac{\partial \phi}{\partial t}\right|_{x, \eta\left(x, t_{n}\right), t}+\left.\frac{1}{2}\left(u^{2}+w^{2}\right)\right|_{x, \eta(x, t), t}+\left.g \eta\right|_{x, t}-\bar{B}
\end{aligned}
$$




\begin{tabular}{ll|ll|ll}
\hline Theory & Order & \multicolumn{2}{|l|}{$H_{1,2}=0.5,1 \mathrm{~m}$} & \multicolumn{2}{|c}{$H_{1,2}=5,10 \mathrm{~m}$} \\
& & $\mu_{K}$ & $\mu_{D}$ & $\mu_{K}$ & $\mu_{D}$ \\
\hline Tadjbakhsh \& Keller (1960) & 3 & 2.99 & 2.00 & 3.00 & 1.96 \\
Goda \& Kakizaki (1966) & 4 & 4.04 & 4.08 & 4.02 & 4.10 \\
Hsu et al (1979) & 3 & 4.00 & 4.00 & 3.98 & 4.00 \\
Present & 5 & 6.00 & 6.01 & 5.97 & 5.98 \\
\hline
\end{tabular}

$h=100 \mathrm{~m}, T=10 \mathrm{~s}$

Table 1: Error exponents for analytical theories.

Both $f_{K}$ and $f_{D}$ should be zero for all $x$ and $t$. But in each case and for each $x$ and $t$, there will be an error, and this error will be proportional to $\varepsilon^{\mu}$. $\varepsilon$ is the expansion parameter adopted by the specific theory; it is related to the wave height. $\mu$ is a dimensionless exponent that measures the accuracy of the specific theory. Specifically, $f_{K} \propto \varepsilon^{\mu_{K}}$ and $f_{D} \propto \varepsilon^{\mu_{D}}$. Evaluating the error at two separate wave heights (but the same water depth and wave period) gives separate expansion parameters $\varepsilon_{1}$ and $\varepsilon_{2}$ and separate error estimates $f_{K 1}, f_{K 2}$ and $f_{D 1}, f_{D 2}$. Accordingly

$$
\frac{f_{K 2}}{f_{K 1}}=\left(\frac{\varepsilon_{2}}{\varepsilon_{1}}\right)^{\mu_{K}}, \quad \frac{f_{D 2}}{f_{D 1}}=\left(\frac{\varepsilon_{2}}{\varepsilon_{1}}\right)^{\mu_{D}}
$$

and

$$
\mu_{K}=\frac{\log \left(f_{K 2} / f_{K 1}\right)}{\log \left(\varepsilon_{2} / \varepsilon_{1}\right)}, \quad \mu_{D}=\frac{\log \left(f_{D 2} / f_{D 1}\right)}{\log \left(\varepsilon_{2} / \varepsilon_{1}\right)}
$$

The dimensionless exponents $\mu_{K}$ and $\mu_{D}$ provide independent fidelity measures of an analytical theory.

Each of the three transitional depth theories above were carefully coded and the $\mu_{K}$ and $\mu_{D}$ exponents computed as field averages for a $50 \times 50$ grid over the range $0 \leq x \leq L / 2$, $0 \leq t \leq T / 2$, where $L$ is the wave length and $T$ is the wave period. The specific wave conditions adopted were depth $h$ of $100 \mathrm{~m}$ and wave period $T$ of $10 \mathrm{~s}$, together with two pairs of wave heights, small $\left(H_{1}=0.5 \mathrm{~m}, H_{2}=1 \mathrm{~m}\right)$ and moderate $\left(H_{1}=5 \mathrm{~m}, H_{2}=10 \mathrm{~m}\right)$ respectively. The results are listed in Table 1.

The third order theory of Tadjbakhsh and Keller (1960) seems accurate to first order only. The fourth order theory of Goda and Kakizaki (1966) seems accurate to third, but not fourth order. An alternative presentation of the same theory (Goda, 1967) does not exactly correspond; a coded version does not perform as well as the Goda and Kakizaki (1966) version. The third order theory of Hsu et al. (1979) does seem to be accurate to third order, a result independently confirmed by Fenton (1985b). It is most probable that typographical errors in the published form of the papers are responsible for the disappointing performance of the earlier theories. 


\section{An Analytical Approach to Fifth Order}

Following the analytical theories cited above for Stokes-style theories for standing waves, it is expected that both the velocity potential function and the water surface elevation can be represented as small parameter perturbation expansions of the form

$$
\phi(x, z, t)=\left(g / k^{3}\right)^{1 / 2} \sum_{i=1}^{N} \varepsilon^{i} \sum_{j=0}^{i} \sum_{m=0}^{i} A_{i j m} \frac{\cosh j k(h+z)}{\cosh j k h} \cos j k x \sin m \omega t
$$

and

$$
\eta(x, t)=\frac{1}{k} \sum_{i=1}^{N} \varepsilon^{i} \sum_{j=0}^{i} \sum_{m=0}^{i} b_{i j m} \cos j k x \cos m \omega t
$$

respectively, in which $h$ is the water depth (see Fig. 1), $k$ is the wave number and $\omega$ is the wave frequency. Equations 10 and 11 have assigned $1 / k$ as the space scale and $(g k)^{-1 / 2}$ as the time scale. $A_{i j m}$ and $b_{i j m}$ are dimensionless coefficients, and $N$ is the order of the analytical theory. Note that the Equation 10 form for the velocity potential function exactly satisfies the field Laplace Equation 1 and the bottom boundary condition (Equation 2 ). Both the $\phi$ and $\eta$ forms also facilitate subsequent imposition of the periodic lateral boundary conditions (Equations 5,16).

The expansion parameter is assigned directly as $\varepsilon=k H / 2$, where $H$ is the standing wave height. This explicit definition was successfully introduced by Isobe and Kraus (1983) in a Stokes-style expansion for progressive waves.

Either the wave number or the wave frequency must also be expanded in terms of $\varepsilon$. As the wave number appears twice in Equation 10 and is included in the adopted space and time scales, it is convenient to expand the wave frequency as

$$
\omega=(g k)^{1 / 2} \sum_{i=1}^{N} \varepsilon^{(i-1)} C_{i}
$$

The Bernoulli constant is expanded as

$$
\bar{B}=\frac{g}{k} \sum_{i=1}^{N} \varepsilon^{i} D_{i}
$$

$C_{i}$ and $D_{i}$ are further dimensionless coefficients.

Given the water depth $h$, the wave height $H$, the wave period $T$, the gravitational acceleration $g$ and the order $\mathrm{N}$ of the theory, the unknowns are the wave number $k$ and the dimensionless coefficients $A_{i j m}, b_{i j m}, C_{i}$ and $D_{i}$. Equations available to define these coefficients are

(1) the kinematic free surface boundary condition (Equation 6)

$$
f_{K}(x, t)=0=\left.\frac{\partial \eta}{\partial t}\right|_{x, t}+\left.\left.u\right|_{x, \eta(x, t), t} \frac{\partial \eta}{\partial x}\right|_{x, t}-\left.w\right|_{x, \eta(x, t), t}
$$


(2) the dynamic free surface boundary condition (Equation 7)

$$
f_{D}(x, t)=0=\left.\frac{\partial \phi}{\partial t}\right|_{x, \eta\left(x, t_{n}\right), t}+\left.\frac{1}{2}\left(u^{2}+w^{2}\right)\right|_{x, \eta(x, t), t}+\left.g \eta\right|_{x, t}-\bar{B}
$$

(3) the periodic lateral boundary conditions

$$
\begin{aligned}
\phi(x, z, t) & =\phi(x+2 \pi / k, z, t)=\phi(x, z, t+2 \pi / \omega) \\
\eta(x, t) & =\eta(x+2 \pi / k, t)=\phi(x, t+2 \pi / \omega)
\end{aligned}
$$

(4) the wave height constraint

$$
f_{H}=0=\eta(0,0)-\eta(L / 2,0)-H \quad \text { OR } \quad f_{H}=0=\eta(0,0)-\eta(0, T / 2)-H
$$

in which the wave length $L=2 \pi / k$ and the wave period $T=2 \pi / \omega$. The options in Equation 17 are identical because of the periodic lateral boundary conditions.

The periodic lateral boundary conditions immediately define that about half the $A_{i j m}$ and $b_{i j m}$ coefficients are zero, specifically those where $j+m$ is an odd integer.

Hyperbolic function quotients appear routinely in the kinematic and dynamic free surface boundary condition equations, through

$$
\begin{aligned}
& \frac{\partial \phi}{\partial t}=\omega\left(g / k^{3}\right)^{1 / 2} \sum_{i=1}^{N} \varepsilon^{i} \sum_{j=0}^{i} \sum_{m=0}^{i} m A_{i j m} \frac{\cosh j k(h+z)}{\cosh j k h} \cos j k x \cos m \omega t \\
u= & \frac{\partial \phi}{\partial x}=-k\left(g / k^{3}\right)^{1 / 2} \sum_{i=1}^{N} \varepsilon^{i} \sum_{j=0}^{i} \sum_{m=0}^{i} j A_{i j m} \frac{\cosh j k(h+z)}{\cosh j k h} \sin j k x \sin m \omega t \\
w= & \frac{\partial \phi}{\partial z}=k\left(g / k^{3}\right)^{1 / 2} \sum_{i=1}^{N} \varepsilon^{i} \sum_{j=0}^{i} \sum_{m=0}^{i} j A_{i j m} \frac{\sinh j k(h+z)}{\cosh j k h} \cos j k x \sin m \omega t
\end{aligned}
$$

The hyperbolic function quotients, evaluated at $z=\eta$ for the free surface boundary conditions become respectively

$$
\begin{aligned}
& \frac{\cosh j k(h+\eta)}{\cosh j k h}=\cosh (j k \eta)+\tanh (j k h) \sinh (j k \eta) \\
& \frac{\sinh j k(h+\eta)}{\cosh j k h}=\tanh (j k h) \cosh (j k \eta)+\sinh (j k \eta)
\end{aligned}
$$

The $\sinh (j k \eta)$ and $\cosh (j k \eta)$ contributions are accommodated through classical series expansions (equivalently Taylor series expansions about the MWL)

$$
\begin{gathered}
\sinh (j k \eta)=j k \eta+\frac{1}{3 !}(j k \eta)^{3}+\frac{1}{5 !}(j k \eta)^{5}+\ldots \\
\cosh (j k \eta)=1+\frac{1}{2 !}(j k \eta)^{2}+\frac{1}{4 !}(j k \eta)^{4}+\ldots
\end{gathered}
$$


in which $\eta$ is represented as Equation 11.

Equations 10 through 13, Equations 18 through 22, and the periodic lateral boundary constraints (Equations 5,16) are used in

(i) the wave height constraint (Equation 17),

(ii) the kinematic free surface boundary condition (Equation 14), and

(iii) the dynamic free surface boundary condition (Equation 15).

Collecting the coefficients of powers of $\varepsilon$ defines the analytical problem at consecutive orders.

At each order there are three algebraic coefficient equations, corresponding to constraints (i) through (iii). The free surface boundary condition constraints, (ii) and (iii), naturally contain powers and products of sine and cosine terms at each order above the first, specifically

(a) at second order:

$\cos ^{2}(\theta), \sin ^{2}(\theta) ; \cos (\theta) \sin (\theta)$

(b) at third order:

$\cos ^{3}(\theta), \sin ^{3}(\theta) ; \cos ^{2}(\theta) \sin (\theta), \sin ^{2}(\theta) \cos (\theta)$

$\cos (2 \theta) \cos (\theta), \cos (2 \theta) \sin (\theta), \sin (2 \theta) \cos (\theta), \sin (2 \theta) \sin (\theta)$

(c) and similar for fourth and fifth order.

$\theta$ is $k x$ or $\omega t$. These power and product terms are expanded as sums of terms like $\cos (\theta), \sin (\theta) ; \cos (2 \theta), \sin (2 \theta) ; \ldots$ using standard trigonometric identities. The free surface boundary condition constraints become the sum of independent terms of the form

$$
a+c f(k x)+\cdots+d g(\omega t)+\ldots+e f(k x) g(\omega t)+\ldots=0
$$

where

- a, c, d and e are coefficient terms,

- $f(k x)$ are trigonometric terms like $\cos (k x), \sin (k x) ; \cos (2 k x), \sin (2 k x) ; \ldots$,

- $g(\omega t)$ are trigonometric terms like $\cos (\omega t), \sin (\omega t) ; \cos (2 \omega t), \sin (2 \omega t) ; \ldots$

As the free surface boundary conditions must be satisfied for all $x$ and $t$, the coefficients terms must also be zero. At each order, the number of unknown coefficients and the number of independent coefficient equations are balanced, leading to a unique solution and the definition of the dimensionless coefficients $A_{i j m}, b_{i j m}, C_{i}$ and $D_{i}$. The coefficient equations are linear, except at first order. Routine use was made of computer algebra software.

The resulting coefficients to fifth order are listed in Appendix A. This fifth order theory was subjected to the same independent numerical confirmation (Richardson extrapolation 
to the limit) as in the previous section for existing analytical theories. The result are listed as the bottom line in Table 1 . The error is of order $\varepsilon^{6}$, so that this theory is clearly correct to fifth order.

As an aide to confirmation of implementation code, a complete list of solution coefficients are given in Appendix B for a specific listed wave condition.

The final step in application of this fifth order theory is the dispersion relationship. In the common situation where $h, H$ and $T$ are known, the dispersion relationship is

$$
(g k)^{1 / 2} \sum_{i=1}^{5} \varepsilon^{(i-1)} C_{i}=2 \pi / T
$$

The left hand side in a function of $k$ (and $h$ and $H$ ), so Equation 24 is a non-linear implicit algebraic equation in $k$, just as in the linear standing wave theory and in linear and nonlinear progressive wave theory. Numerical solution for $k$ is a routine problem in numerical analysis, with robust algorithms in any reasonable engineering software platform. Where $L=2 \pi / k$ rather than $T=2 \pi / \omega$ is given, the solution for $\omega$ is explicit, from Equation 12 .

\section{$5 \quad$ Nonlinear Kinematics}

Existing presentations of higher-order standing wave theory are extremely sparse on demonstration of the non-linear features of the predictions. A sequence of comparisons are presented here to highlight the significant non-linear aspects of the present fifth-order theory. Each example shows the non-linear predictions together with the linear theory, where the velocity potential function, the water surface elevation and the dispersion relationship are respectively

$$
\begin{gathered}
\phi(x, z, t)=-\frac{a g}{\omega} \frac{\cosh k(h+z)}{\cosh k h} \cos k x \sin \omega t \\
\eta(x, t)=a \cos k x \cos \omega t \\
\omega^{2}=g k \tanh k h
\end{gathered}
$$

in which the wave amplitude $a$ is $H / 2$,

The specific wave conditions used for comparison are those for which the solution coefficients are listed in Appendix B, namely water depth $h$ of $100 \mathrm{~m}$, wave height $H$ of $10 \mathrm{~m}$ and period $T$ of $10 \mathrm{~s}$. For the fifth order theory, the wave number $k$ is $0.04068 \mathrm{~m}^{-1}$ (and wave length $L$ is $154.45 \mathrm{~m}$ ). For the linear theory, the wave number $k$ is $0.04027 \mathrm{~m}^{-1}$ (and wave length $L$ is $156.03 \mathrm{~m}$ ).

Figure 2 shows the water surface profiles $\eta(x ; t)$ over a half wave length at time intervals of $0.5 \mathrm{~s}(\Delta t / T=0.05)$. The left-hand plot, Figure 2a, is the fifth-order theory and the right-hand plot, Figure 2b, is the linear theory. One immediately apparent feature of the non-linear prediction is the expected super-elevation of the crest (to $5.498 \mathrm{~m}$ ) and the corresponding flattening of the trough (to $-4.502 \mathrm{~m})$. This is a routine feature also of nonlinear progressive waves. Another obvious feature is the periodic-in- $x$ translation of the non-linear zero-crossing position over a horizontal range of about $5 \mathrm{~m}$. 


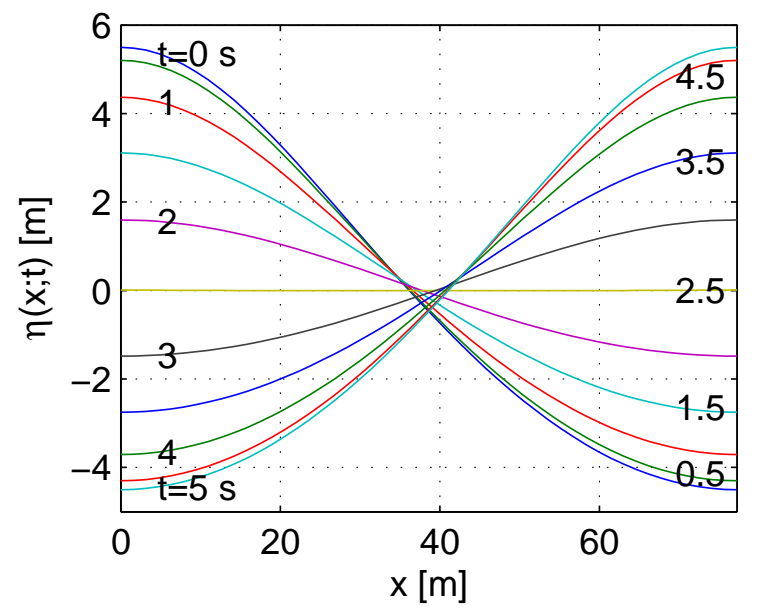

(a) 5th order

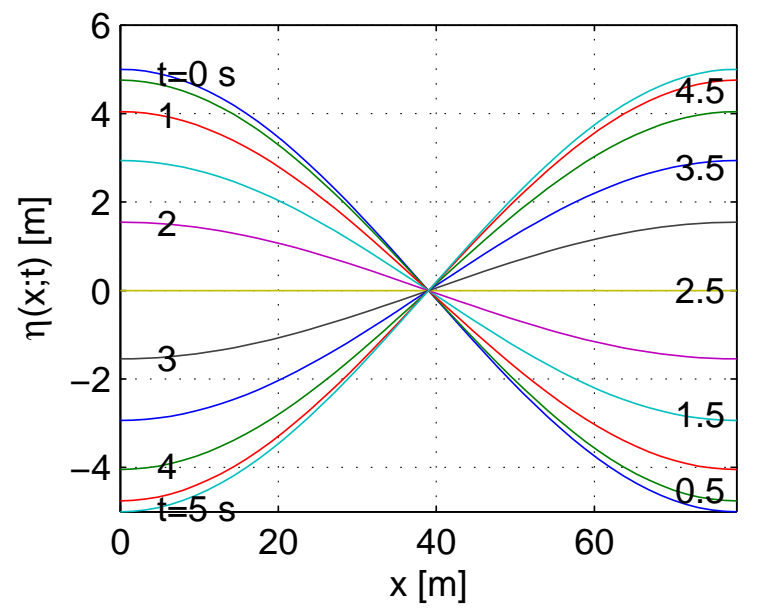

(b) Linear

Figure 2: Water surface profiles, $\eta(x ; t)$

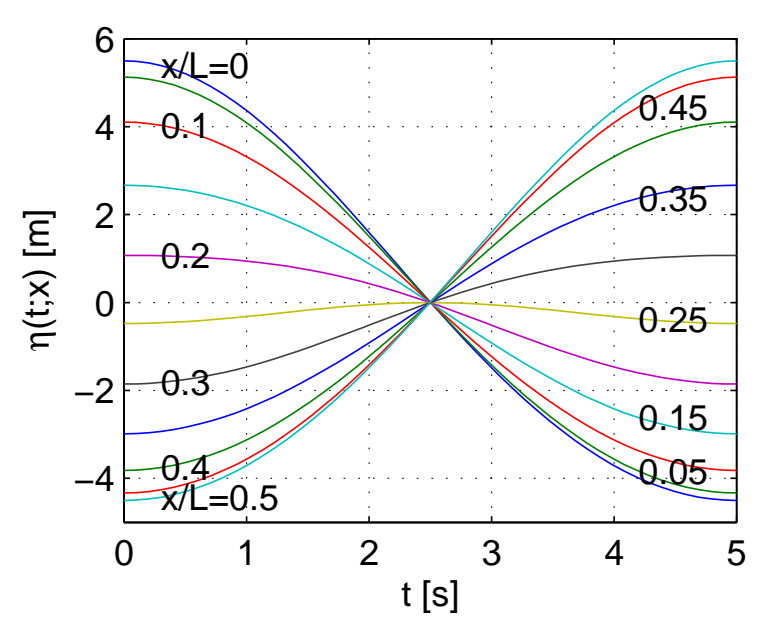

(a) 5th order

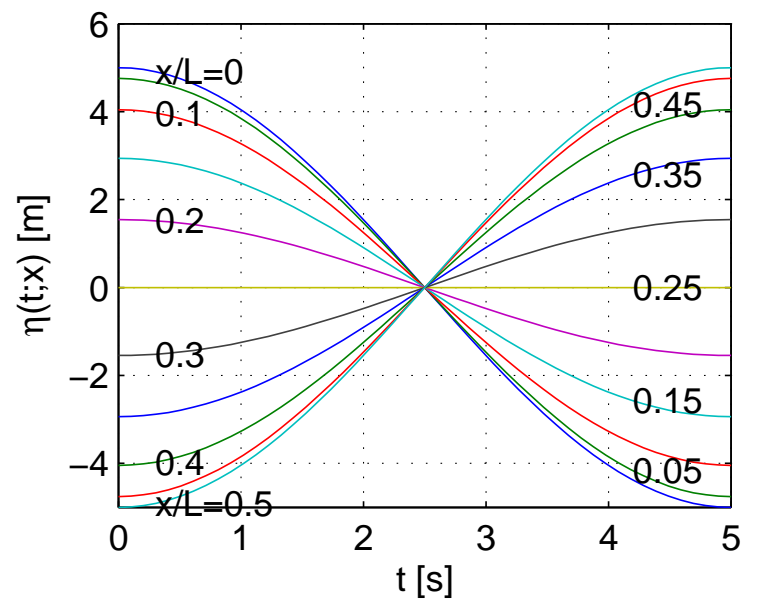

(b) Linear

Figure 3: Water surface time histories, $\eta(t ; x)$

Figure 3 shows the water surface time history $\eta(t ; x)$ over a half wave period at spatial intervals of $\Delta x / L=0.05$. There is no periodic-in- $t$ translation of the zero-crossing time, and the traces otherwise reflect the crest super-elevation and trough flattening.

The vertical acceleration is

$$
\frac{D w}{D t}=\frac{\partial w}{\partial t}+u \frac{\partial w}{\partial x}+w \frac{\partial w}{\partial z}
$$

The horizontal velocity $u$ and the vertical velocity $w$ are given by Equations 19 and 20 respectively. The additional time and space partial derivatives required in Equation 26 following from differentiation. Figure 4 shows vertical profiles of the vertical acceleration $D w / D t(z ; x=0, t)$ at a fixed position $x$ position $(x=0)$ at time intervals of $0.5 \mathrm{~s}(\Delta t / T$ 


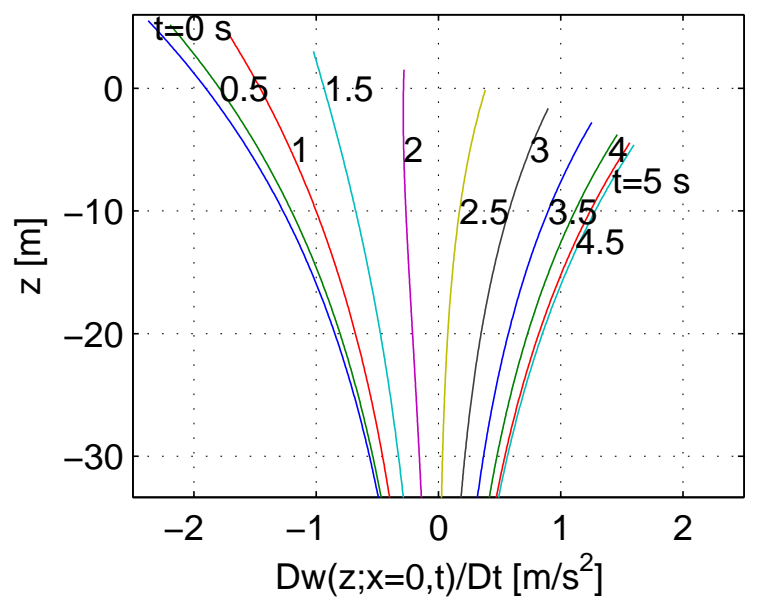

(a) 5 th order

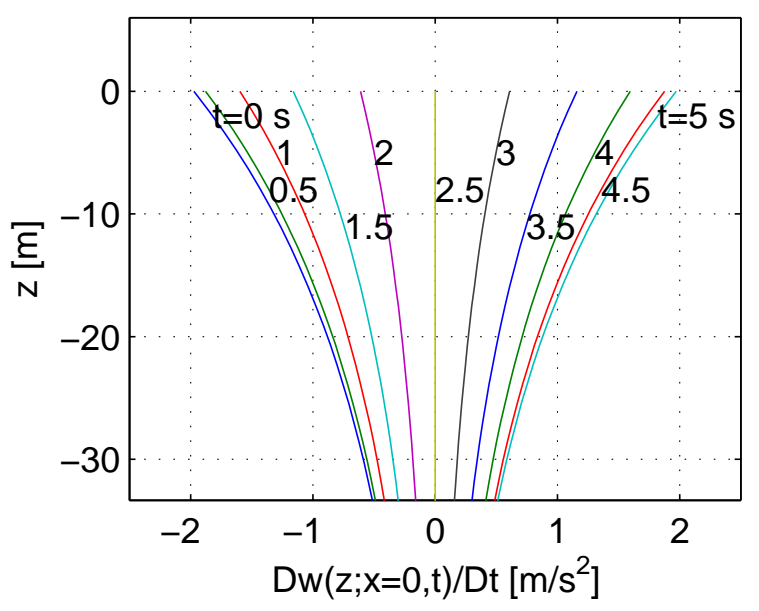

(b) Linear

Figure 4: Vertical acceleration profiles, $D w(z ; x=0, t) / D t$

$=0.05)$. The expected increase in the magnitude is clear in both (a) and (b). The major difference is in the advanced phase of the vertical profiles from the non-linear prediction at times in the general neighbourhood of $T / 4$. Again, this pattern reflects the crest superelevation and trough flattening.

The dynamic pressure is

$$
\frac{p_{d}}{\rho}=\bar{B}-\frac{\partial \phi}{\partial t}-\frac{1}{2}\left(u^{2}+w^{2}\right)
$$

where the component terms use Equations 13, 18, 19 and 20 respectively. Figure 5 shows horizontal profiles of the dynamic pressure $p_{d}(x ; z=-h / 3, t)$ at a fixed elevation $(z=$ $-h / 3)$ at time intervals of $0.5 \mathrm{~s}(\Delta t / T=0.05)$. It reveals an interesting pattern. The non-linear profiles show the expected crest-trough asymmetry but reverse the pattern in Figure 2. For $\eta(x ; t)$, the crest amplitude (at $\eta(0,0)$ or $\eta(0.5 L, 0.5 T)$ ) exceeds the trough amplitude $(\eta(0,0.5 T)$ or $\eta(0.5 L, 0))$. For $p_{d}(x ; t)$, the trough amplitude exceeds the crest amplitude in magnitude. Additionally, the crest amplitude is no longer at $(x, t)=(0,0)$ or $(0.5 L, 0.5 T)$ but has moved closer in time to the quarter period, as approximately $(0,0.15 T)$ and $(0.5 L, 0.35 T)$. The timing of the crest varies with the elevation $z$.

Figure 6 shows the dynamic pressure time history $p_{d}(t ; x, z=-h / 3)$ at a fixed elevation $(z=-h / 3)$ over a half wave period at spatial intervals of $\Delta x / L=0.05$. The pattern anticipated in Figure 5 is again apparent here. The trough amplitude is larger in magnitude, with the timing of the crest again no longer at $(x, t)=(0,0)$ or $(0.5 L, 0.5 T)$ but closer to the quarter period time. The Figure 6a prediction has been anticipated by Goda (1967) in an investigation of sea bed pressures under standing waves.

As a supplementary illustration of predicted kinematics, Figure 7 shows selected kinematics in (a) shallow transitional $\left(h_{1}=15 \mathrm{~m}, \omega^{2} h_{1} / g=0.60\right)$ and (b) deep water $\left(h_{2}=100 \mathrm{~m}\right.$, $\left.\omega^{2} h_{2} / g=4.02\right)$. In both cases, the wave height $H$ is $5 \mathrm{~m}$ and the wave period $T$ is $10 \mathrm{~s}$. The 


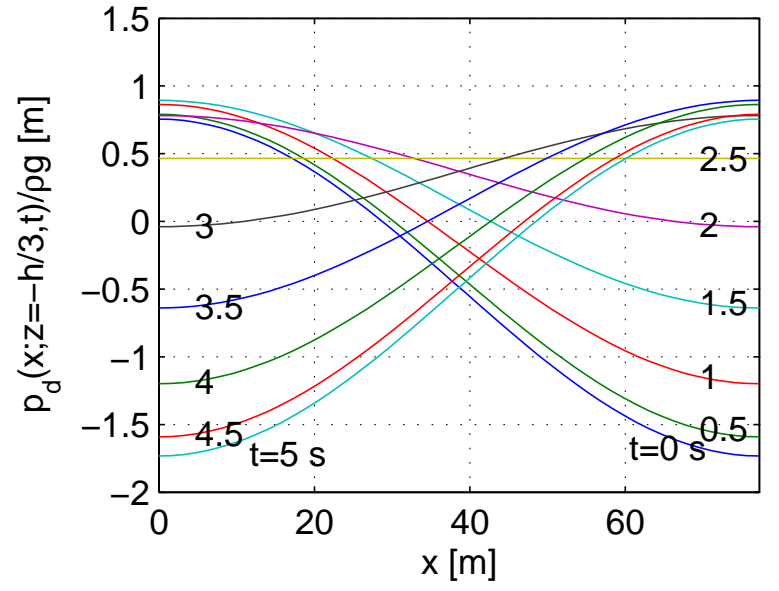

(a) 5th order

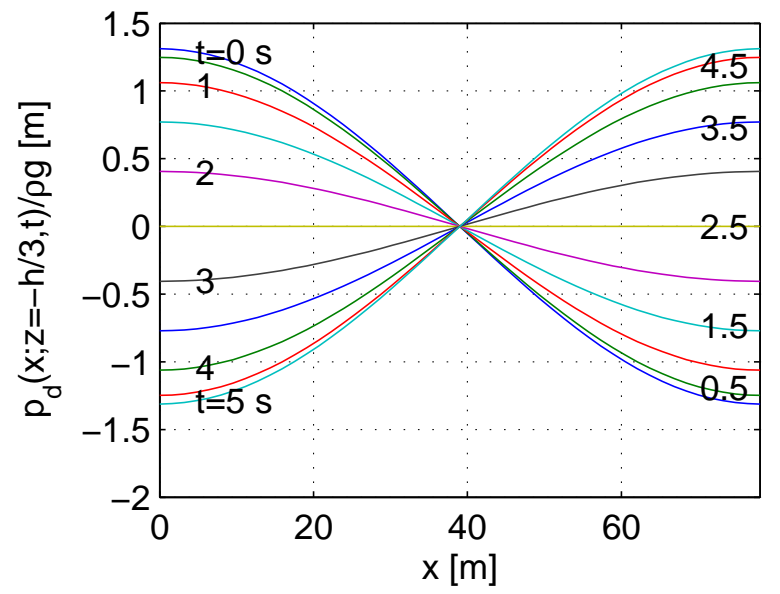

(b) Linear

Figure 5: Dynamic pressure profiles, $p_{d}(x ; z=-h / 3, t)$

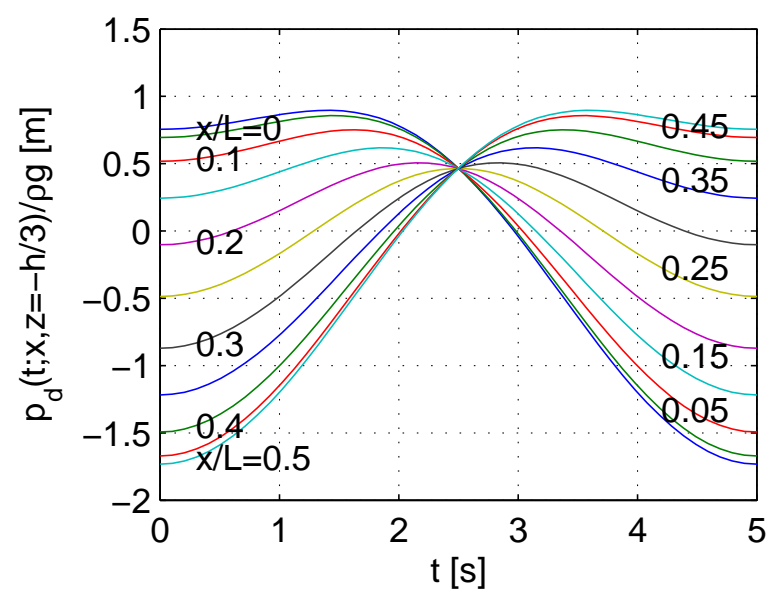

(a) 5th order

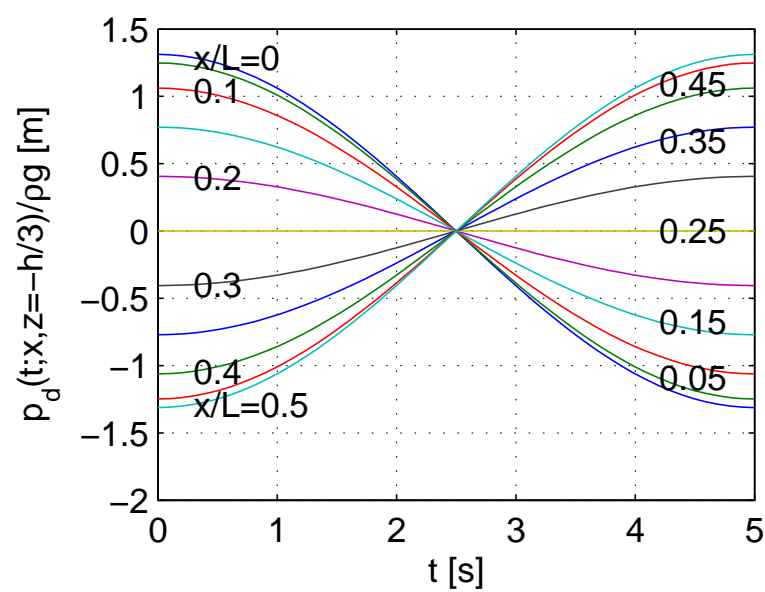

(b) Linear

Figure 6: Dynamic pressure time histories, $p_{d}(t ; x, z=-h / 3)$

top line shows the water surface profiles $\eta(x ; t)$ at times $t=0$ and $t=T / 2$. These profiles show the expected response. The wave length is rather shorter in the shallow transitional case (a), where there is also rather move crest superelevation and trough flattening.

The middle line shows the vertical profiles of the vertical acceleration $D w(z ; x=$ $0, t) / D t$ at $x=0$ and at the same two times. The notable feature here is the enhanced magnitude of the vertical acceleration under the crest in (a), which follows from the general sharpening of kinematic activity under the crest.

The bottom line shows the time history of the dynamic pressure $p_{d}\left(t ; z=-h_{1}, x\right)$ at the bed $z=-h_{1}$ for (a) and at the same depth for (b), both at horizontal positions $x=0$ and $x=L / 2$. The bias toward the trough amplitude, identified in Figures 5 and 6 , is again apparent, though the extent is reduced by the smaller wave height. 

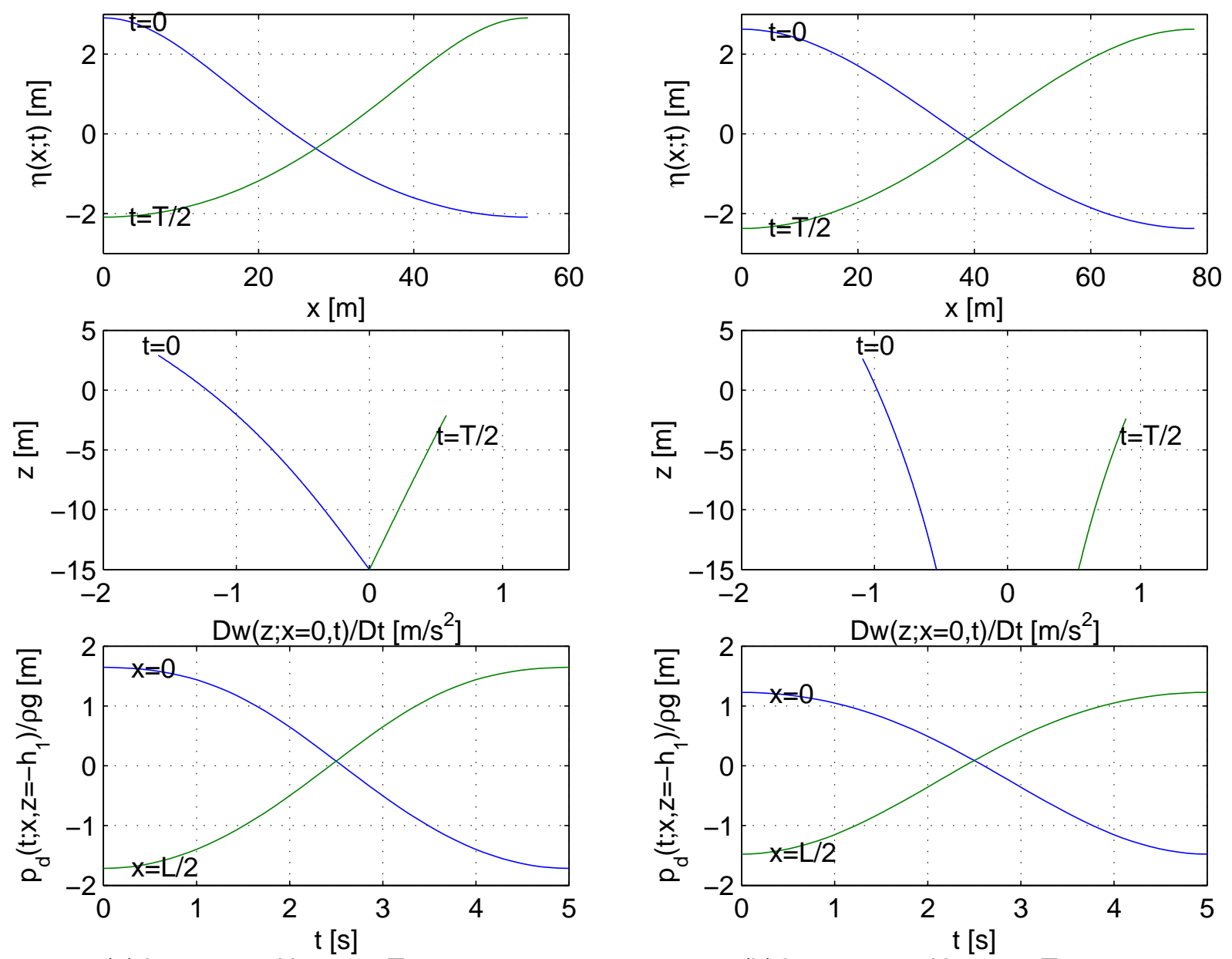

(a) $\mathrm{h}_{1}=15 \mathrm{~m} ; \mathrm{H}=5 \mathrm{~m}, \mathrm{~T}=10 \mathrm{~s}$

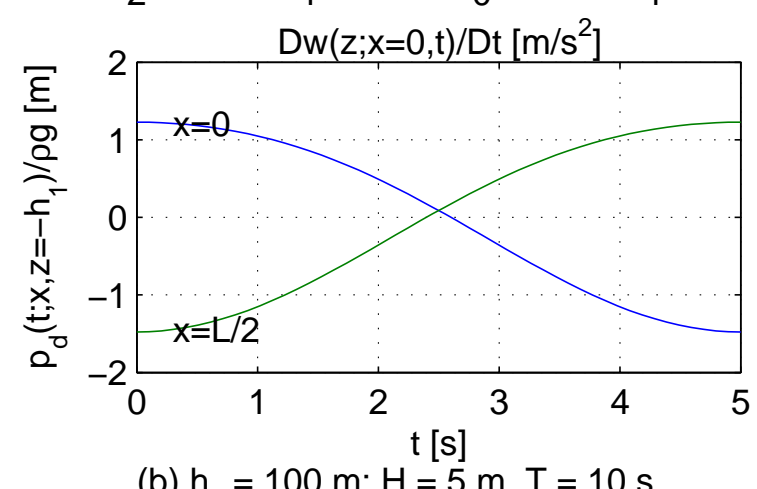

Figure 7: Comparison of selected kinematics in (a) shallow transition and (b) deep water

\section{Range of validity}

From the experience with non-linear Stokes-style theories for progressive waves, there is some expectation that the limit of validity may be restricted to waves of moderate height in deeper water. There is no strong expectation that the precision will extend close to the limit wave, or that the Stokes-style theory will extend very far into shallow water.

The limit wave is expected to have a slope $\partial \eta / \partial x$ discontinuity at the crest. Equation 11 for the water surface is smooth and continuous, precluding a crest discontinuity. The dominant length scales for shallow water (cnoidal) progressive waves are $h$ and $H$, whereas they are $L$ (or $1 / k)$ and $H$ in the Stokes-style deep water theory. But it is also known (e.g. Williams, 1985) that very high order Stokes-style theories can push these limits somewhat.

The limits of the present fifth-order theory are now investigated, over a range of dimensionless depths $\omega^{2} h / g$ from 0.6 (shallow-transitional) to 4.0 (deep) and a range of 


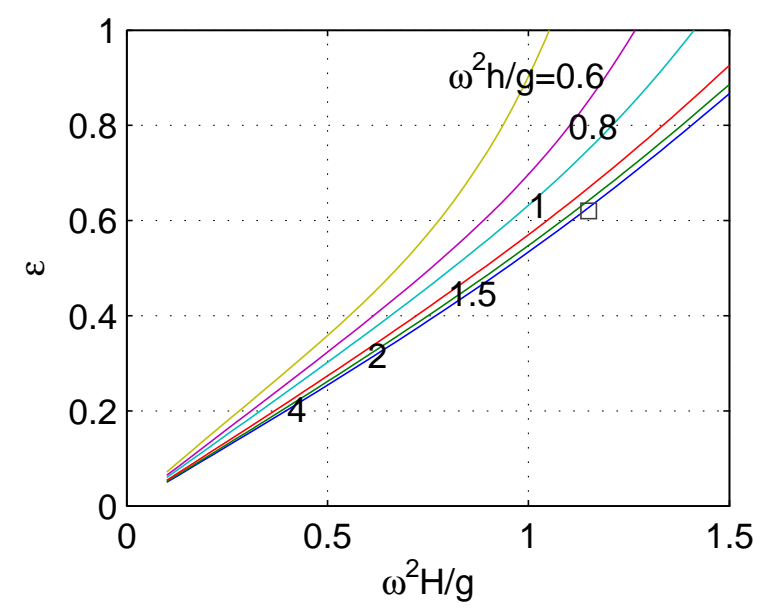

(a) Expansion Parameter

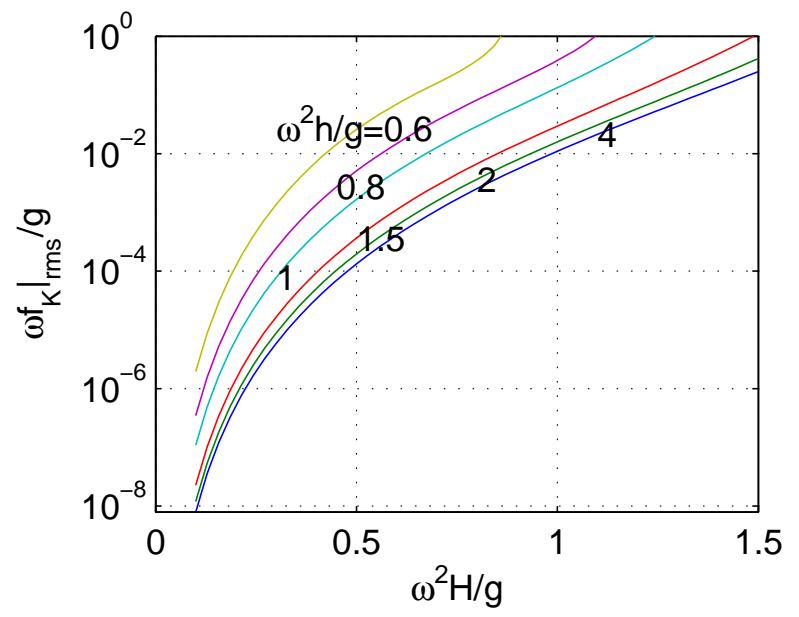

(b) rms Error in KFSBC

Figure 8: Evolution of $\varepsilon$ and rms Error in KFSBC

dimensionless wave heights $\omega^{2} H / g$ from 0.1 to 1.5. There is no expectation that the limit standing wave will reach $\omega^{2} H / g=1.5$. For progressive waves, $\omega^{2} H / g$ reaches an asymptotic maximum of 1.0575 in deep water (Williams, 1985), falling to about 0.4 at $\omega^{2} h / g=0.6$.

Figure 8a shows the evolution of the expansion parameter $\varepsilon$ for standing wave conditions over the range of dimensionless depth and dimensionless wave height considered. The analytical fifth-order theory is based on small parameter perturbation expansions for $\phi$, $\eta, \omega$ and $\bar{B}$, with each order being a small correction to the previous order. Expansion parameters significantly in excess of about 0.5 would seem to be stretching the definition of small correction too far. This situation is reached at wave heights $\omega^{2} H / g$ approaching 1 in deep water and at progressively lower wave heights in shallower water. For deep water (theoretically $\omega^{2} h / g=\infty$, but pragmatically $\omega^{2} h / g \gtrsim 2.5$ ), Mercer and Roberts (1992) predict that the limit standing wave steepness (defined as the present expansion parameter) is 0.6202 , which corresponds to a deep water limit wave of $\omega^{2} H / g \approx 1.14$. This limit wave prediction is the box marker in Figure 8a. Transitional water limit waves would not be expected to reach this $\omega^{2} \mathrm{H} / \mathrm{g}$ limit.

Figure $8 \mathrm{~b}$ addresses the dimensionless rms error in the KFSBC, estimated from Equation 14 over a $50 \times 50$ grid extending over $0 \leq x \leq L / 2$ and $0 \leq t \leq T / 2$. The dimensionless rms error in the DFSBC provides an almost identical result. The consequences of the Figure $8 \mathrm{a}$ trend to expansion parameters that are no longer small is now much clearer. There is a gradual transition to progressively larger KFSBC error in shallower water. These integral measures of the solution in Figures $8 \mathrm{a}$ and b suggest a progressive loss of validity for the more extreme waves in deep water and for even small to moderate waves in shallower water.

Predicted field variables often provide a more explicit measure of suspect validity, and routine perusal of field solution surfaces for $\eta, u, w, D u / D t, D w / D t$ and $p_{d}$ is always a 


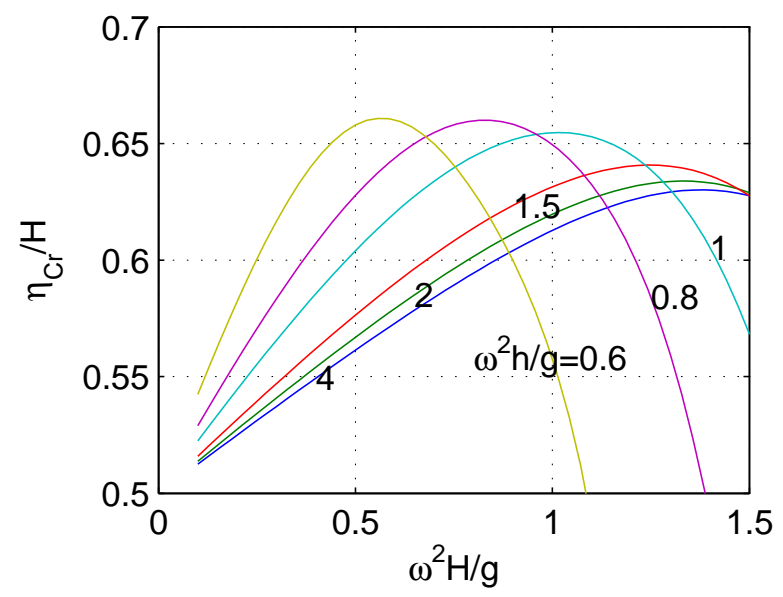

(a) Crest Elevation

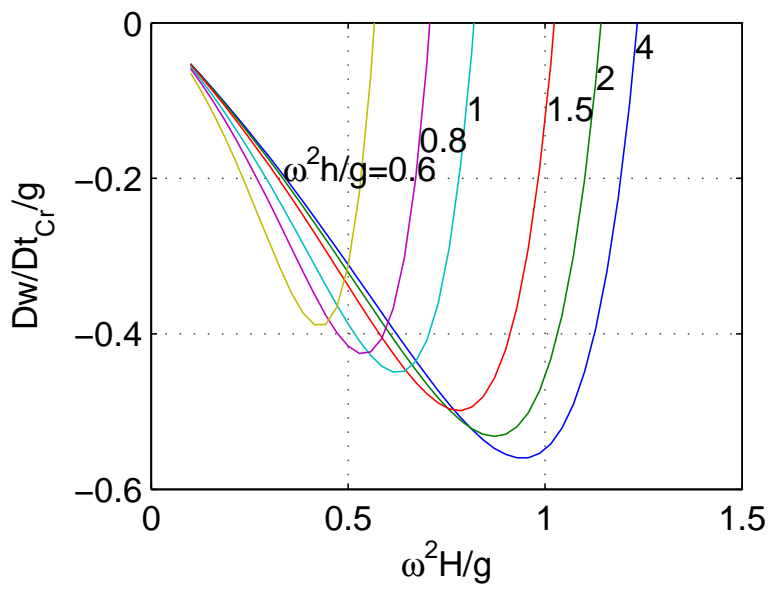

(b) Vertical Acceleration at Crest

Figure 9: Evolution of Crest Elevation and Crest Acceleration

good practice. For progressive waves, early warning of suspect validity is often provided by a lack of smoothness in the water surface profile. For the present standing wave theory, extensive exploration of solution surfaces suggested other aspects of the field solution as early indicators of predictive difficulties.

The more focused kinematic measures in Figures 9a and b provide a much more explicit view of the limits of validity. Figure 9 a addresses the crest elevation $\eta(0,0) / H$. Experience with non-linear progressive waves, together with the more limited experience for non-linear standing waves (see Figures 2 and 3), suggest that the crest elevation climbs above $0.5 \mathrm{H}$, increasing with increasing wave height. That is indeed observed in Figure 9a for standing waves in deep water for wave heights to $\omega^{2} H / g \approx 1$. For wave heights beyond $\omega^{2} H / g \approx 1$, these deep water standing wave predictions reach a maximum and begin to decline. This is very unlikely to happen and suggests that this behaviour is a sign of limiting validity. This maximum and trend reversal is well beyond $\omega^{2} H / g \approx 1$ where the wave may indeed be beyond the limit wave. The shallower water predictions shift this maximum and trend reversal to significantly smaller wave heights.

The vertical acceleration $D w / D t$ at the crest is negative and its approach to the gravitational acceleration $\vec{g}$ has been used (Penney and Price, 1952; Schwartz and Whitney, $1981)$ as an indicator of the limit wave. Predictions of the evolution of the crest acceleration $(D w(0,0) / D t) / g$ are shown in Figure $9 \mathrm{~b}$, and reveal a pattern very similar in concept to the crest elevation in Figure 9a, but more extreme. With increasing wave height, the crest acceleration initially decreases (increases in magnitude) toward $\vec{g}$, but reaches a minimum well before the gravitational acceleration is approached. And this minimum moves even further from the gravitational acceleration as the water shallows. Beyond the minimum, the vertical acceleration rapidly goes positive, a prediction that has no physical credibility.

The maxima in the crest elevation (Figure 9a) and the minima in the vertical acceleration at the crest (Figure 9b) are strong indicators of the limits of validity of the present 


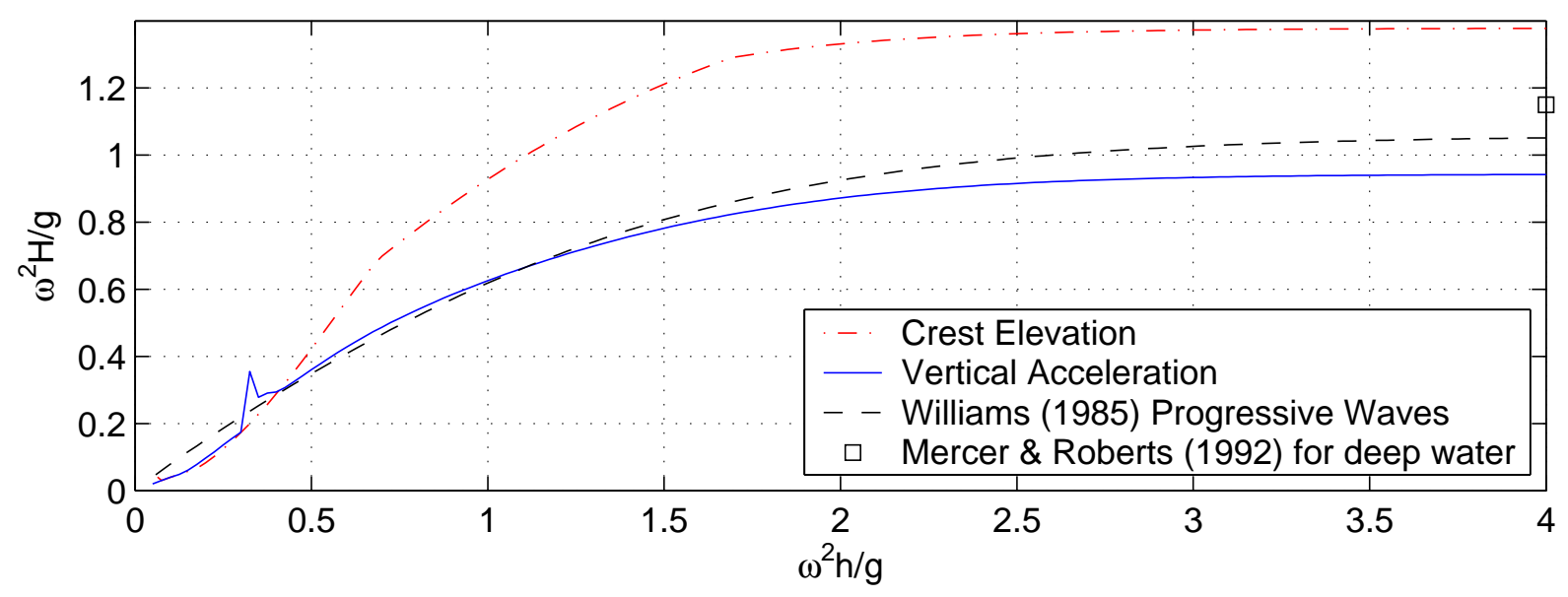

Figure 10: Indicators of Limit Standing Wave

fifth order theory, though perhaps beyond the limits. The evolution of these stationary points for crest elevation $\eta_{C r} / H$ (where $\eta_{C r}=\eta(0,0)$ ) and crest acceleration $D w / D t_{C r} / g$ (where $D w / D t_{C r}=D w\left(0, \eta_{C r}, 0\right) / D t$ ) were pursued numerically as a problem in nonlinear optimisation. In a single unknown, this is a standard problem in numerical analysis with robust algorithms in any reasonable engineering software platform. Stationary point solutions (for $\omega^{2} H / g$ ) were sought for a range of $\omega^{2} h / g$ depths from 0.05 to 4.0.

The evolution of these stationary point estimates with water depth are presented in Figure 10. The trend in the evolution with depth is expected. Higher waves can be expected in deeper water, to a plateau level that does nor further increase with depth. Progressively smaller limits are expected in shallower water, where eventually the Stokesstyle expansion must break down. This can be seen in the erratic predictions for $\omega^{2} h / g \lesssim$ 0.5 .

Their relative magnitudes were anticipated from Figure 9 and the vertical acceleration provides the closest the present analytical theory can come to a pragmatic measure of the limits of validity. While the limit of validity is expected to be associated with an approach to limit wave conditions, there is no suggestion that this limit of validity is also an indicator of limit wave conditions. Indeed, Figure 9b suggests that the present analytical theory has the vertical acceleration $D w / D t$ reaching only about $-0.55 g$ in deep water, whereas Mercer and Roberts (1992) predict $-0.98 g$ for the limit wave in deep water.

Nevertheless, a comparison of the trend of this limit of validity with the trend of limit wave predictions for progressive waves is intriguing. For progressive waves, Williams (1985) provides tabulated predictions of the limit wave height for twenty-two $\omega^{2} h / g$ depths between 0.06 and 12.0. The theory is Stokes-style and computer extended to very high orders. A rational approximation to these tabulated predictions is

$$
\left.\frac{\omega^{2} H_{\text {limit }}}{g}\right|_{\text {Progressive }}=c_{0} \tanh \frac{a_{1} p+a_{2} p^{2}+a_{3} p^{3}}{1+b_{1} p+b_{2} p^{2}}
$$

where $p=\omega^{2} h / g, a_{1}=0.7879, a_{2}=2.0064, a_{3}=-0.0962, b_{1}=3.2924, b_{2}=-0.2645$ and 
$c_{0}=1.0575$ has a maximum error of 0.0014 over the range of the tables.

This prediction is also included on Figure 10. And it closely tracks the trend of the vertical acceleration prediction for the limit of validity for the present analytical theory. The asymptotic deep water limit for the vertical acceleration prediction is approximately 0.94. This is expected to underpredict limit wave conditions. As discussed in relation to Figure 8a, Mercer and Roberts (1992) predict a deep water limit standing wave of $\omega^{2} H / g \approx 1.14$. This point is included on Figure 10, again as a box marker on the right. A preliminary estimate of the limit standing wave would be Equation 28 with $c_{0} \approx 1.14$ (instead of 1.0575).

Extension of this analysis to higher order and specific attention to shallower water conditions would be necessary to further refine this estimate. A numerical approach with this objective is in progress.

Note also that the moderate wave condition adopted for discussion in Section 5 and Appendix B has $\omega^{2} h / g=4.02$ and $\omega^{2} H / g=0.40$, which is well within the suggested limits of validity.

\section{Conclusions}

Existing analytical theories for standing waves follow almost exactly the same analytical formulation. Unfortunately, the predictive equations are inconsistent, no doubt attributable to typographical errors. They are also algebraically long and complicated, and so difficult to reconcile. The relative complexity coupled with this typographical uncertainty does not encourage engineering application. The fidelity of the published form of each of these existing theories can be numerically demonstrated from Richardson extrapolation to the limit. The single third-order theory that escapes typographical presentation compromise is identified.

A new analytical theory to fifth order is outlined. The expansion parameter is direct, $k H / 2$, so that there is only a single unknown $k$ in the dispersion relationship. Richardson extrapolation to the limit confirms that this theory is indeed correct to fifth order. A numerical listing of the numerous theory coefficients for a specific wave condition is provided in an appendix, to facilitate development of application code.

Existing presentations of the non-linear kinematics in standing waves are sparse and attention is given to the identification of specifically non-linear aspects of the kinematics response. A side-by-side focus on predicted profiles and time histories of the water surface $\eta$, the vertical acceleration $D w / D t$ and the dynamic pressure $p_{d}$, from both the fifth order theory and the linear theory, provide a detailed picture (Figures 2 through 6) of the expected response patterns.

Finally, attention is directed to the limits of applicability of the present analytical theory. It is shown (Figure 8) that integral properties of the solution, specifically the expansion parameter $\varepsilon=k H / 2$ and the rms error in the free surface boundary conditions, evolve to apparently unacceptable levels as the dimensional wave height $\omega^{2} H / g$ increases toward and beyond unity, and as the dimensional water depth $\omega^{2} h / g$ shallows. Among 
local solution variables, the relative crest elevation, $\eta_{C r} / H$, reaches a predicted maximum and the vertical acceleration at the crest $D w / D t_{C r}$ reaches a predicted minimum (Figure 9). Physically, these predictions are questionable.

Further pursuit of these stationary points identifies the vertical acceleration at the crest as an appropriate indicator of the limits of validity of the present analytical theory (Figure 10). A comparison of the trend curve for the minimum of the vertical acceleration at the crest with limit wave predictions for progressive waves suggests a pragmatic preliminary estimate for the trend of limit wave height in standing waves.

\section{A Coefficients to Fifth Order}

$C_{i}, D_{i}$ and non-zero $A_{i j k}$ and $b_{i j k}$ coefficients.

$q=\tanh (k h)$

\section{A.1 First Order}

$C_{1}=\sqrt{q}, \quad D_{1}=0, \quad A_{1,1,1}=-\frac{1}{\sqrt{q}}, \quad b_{1,1,1}=1$

\section{A.2 Second Order}

$$
\begin{aligned}
& C_{2}=0, \quad D_{2}=-\frac{1}{8} \frac{q^{2}-1}{q}, \quad A_{2,2,2}=\frac{3}{16} \frac{-1+q^{4}}{q^{7 / 2}}, \quad A_{2,0,2}=\frac{1}{16} \frac{1+3 q^{2}}{q^{3 / 2}} \\
& b_{2,2,0}=\frac{1}{8} \frac{1+q^{2}}{q}, \quad b_{2,2,2}=-\frac{1}{8} \frac{q^{2}-3}{q^{3}}
\end{aligned}
$$

\section{A.3 Third Order}

$C_{3}=-\frac{1}{64} \frac{-9+3 q^{4}+12 q^{2}+2 q^{6}}{q^{7 / 2}}, \quad D_{3}=0$

$A_{3,1,3}=-\frac{1}{256} \frac{31 q^{4}-62 q^{2}-9}{q^{9 / 2}}, \quad A_{3,1,1}=\frac{1}{256} \frac{6 q^{10}+11 q^{8}-63 q^{6}+96 q^{4}+27 q^{2}+27}{q^{13 / 2}}$

$A_{3,3,3}=-\frac{1}{256} \frac{39 q^{6}-53 q^{4}+5 q^{2}+9}{q^{13 / 2}}, \quad A_{3,3,1}=-\frac{1}{256} \frac{6 q^{8}-13 q^{6}-5 q^{4}+9 q^{2}+3}{q^{9 / 2}}$

$b_{3,1,3}=\frac{1}{256} \frac{5 q^{4}-18 q^{2}-3}{q^{4}}, \quad b_{3,1,1}=-\frac{1}{256} \frac{72 q^{4}+6 q^{10}+3 q^{8}-43 q^{6}+15 q^{2}+27}{q^{6}}$

$b_{3,3,3}=-\frac{3}{256} \frac{-3 q^{4}+q^{6}+3 q^{2}-9}{q^{6}}, \quad b_{3,3,1}=\frac{3}{256} \frac{27 q^{2}+2 q^{8}+q^{6}-15 q^{4}+9}{q^{4}}$

\section{A.4 Fourth Order}

$C_{4}=0$

$D_{4}=\frac{1}{1024} \frac{39 q^{4}-56 q^{8}+87 q^{6}+5 q^{10}-72 q^{2}+6 q^{12}-9}{q^{7}}$ 


$$
\begin{aligned}
& A_{4,4,4}=\frac{1}{12288} \frac{197 q^{12}+1732 q^{10}+1481 q^{8}-9872 q^{6}+7623 q^{4}-756 q^{2}-405}{\left(q^{2}+5\right) q^{19 / 2}} \\
& A_{4,2,4}=\frac{1}{3072} \frac{398 q^{10}+63 q^{8}-1298 q^{6}+144 q^{4}+1188 q^{2}+81}{\left(4 q^{2}+3\right) q^{15 / 2}} \\
& A_{4,2,2}=\frac{1}{3072} \frac{18 q^{12}+259 q^{10}-240 q^{8}-256 q^{6}+252 q^{4}+189 q^{2}+162}{q^{19 / 2}} \\
& A_{4,4,2}=\frac{1}{6144} \frac{54 q^{14}+207 q^{12}-1060 q^{10}-1743 q^{8}+4502 q^{6}-207 q^{4}-648 q^{2}-81}{\left(3+q^{2}\right) q^{15 / 2}} \\
& b_{4,2,0}=-\frac{1}{1024} \frac{27 q^{4}+18 q^{8}-23 q^{6}+32 q^{10}+63 q^{2}+27}{q^{7}} \\
& b_{4,2,2}=-\frac{1}{768} \frac{90 q^{4}+81+54 q^{2}-68 q^{6}+45 q^{8}+54 q^{10}}{q^{9}} \\
& b_{4,4,2}=-\frac{1}{768} \frac{-81+288 q^{4}-621 q^{2}+518 q^{6}-273 q^{8}-105 q^{10}+18 q^{12}}{q^{7}\left(3+q^{2}\right)} \\
& b_{4,2,4}=-\frac{1}{3072} \frac{81-351 q^{4}+1053 q^{2}+283 q^{6}+6 q^{8}}{q^{7}\left(4 q^{2}+3\right)} \\
& b_{4,4,4}=\frac{1}{3072} \frac{522 q^{4}+405+81 q^{2}-262 q^{6}+q^{8}+21 q^{10}}{q^{9}\left(q^{2}+5\right)} \\
& A_{4,0,2}=-\frac{1}{2048} \frac{-122 q^{4}-91 q^{8}+344 q^{6}+27 q^{10}+117 q^{2}+18 q^{12}+27}{q^{15 / 2}} \\
& b_{4,4,0}=\frac{1}{1024} \frac{66 q^{4}-66 q^{8}+2 q^{6}+21 q^{10}+81 q^{2}+6 q^{12}+18}{q^{7}} \\
& A_{4,0,4}=\frac{1}{4096} \frac{-185 q^{4}+67 q^{8}-235 q^{6}+207 q^{2}+18}{q^{12}}
\end{aligned}
$$

\section{A.5 Fifth Order}

$$
\begin{aligned}
& C_{5}=-\frac{1}{16384}\left(12 q^{16}-176 q^{14}-681 q^{12}+201 q^{10}+279 q^{8}-978 q^{6}-279 q^{4}\right. \\
& \left.\quad+513 q^{2}+405\right) /\left(q^{19 / 2}\right) \\
& D_{5}=0 \\
& A_{5,5,1}=-\frac{1}{65536}\left(180 q^{22}-360 q^{20}-1329 q^{18}-1389 q^{16}-10180 q^{14}+25076 q^{12}\right. \\
& \left.\quad+40794 q^{10}-62542 q^{8}+3864 q^{6}+3132 q^{4}+2511 q^{2}+243\right) \\
& \quad /\left(q^{21 / 2}\left(3+q^{2}\right)\left(5 q^{2}+3\right)\right) \\
& A_{5,1,3}=\frac{1}{65536}\left(192 q^{16}-8536 q^{14}-24494 q^{12}-2281 q^{10}-7833 q^{8}-34170 q^{6}\right. \\
& \left.\quad-16344 q^{4}-25461 q^{2}-2673\right) /\left(q^{21 / 2}\left(4 q^{2}+3\right)\right) \\
& A_{5,5,3}=-\frac{1}{65536}\left(10290 q^{22}+43395 q^{20}-173337 q^{18}-602962 q^{16}+701240 q^{14}\right. \\
& \left.\quad+1757970 q^{12}-1345366 q^{10}-1223160 q^{8}+844206 q^{6}+21555 q^{4}-31401 q^{2}-2430\right) \\
& \quad /\left(q^{21 / 2}\left(q^{4}+8 q^{2}+15\right)\left(5 q^{4}+5 q^{2}-2\right)\right) \\
& A_{5,5,5}=-\frac{1}{65536}\left(5415 q^{16}+32830 q^{14}-2142 q^{12}-121450 q^{10}+28240 q^{8}+135290 q^{6}\right.
\end{aligned}
$$




$$
\begin{aligned}
& \left.-88578 q^{4}+8370 q^{2}+2025\right) /\left(q^{25 / 2}\left(q^{2}+5\right)\left(3 q^{2}+5\right)\right) \\
& A_{5,1,5}=-\frac{1}{65536}\left(1828 q^{12}+352 q^{10}-10757 q^{8}+35788 q^{6}-594 q^{4}-8100 q^{2}-405\right) \\
& /\left(q^{21 / 2}\left(4 q^{2}+3\right)\right) \\
& A_{5,3,3}=-\frac{1}{65536}\left(5400 q^{22}+12918 q^{20}-137093 q^{18}-272731 q^{16}+357403 q^{14}\right. \\
& \left.+299725 q^{12}+100929 q^{10}+467859 q^{8}+215865 q^{6}-91935 q^{4}-169128 q^{2}-43740\right) \\
& /\left(\left(35 q^{4}+84 q^{2}+45+4 q^{6}\right) q^{25 / 2}\right) \\
& A_{5,1,1}=\frac{1}{65536}\left(2259900+17432820 q^{2}-119464668 q^{6}+24483303 q^{4}\right. \\
& -1317006294 q^{14}-99942680 q^{26}-468613281 q^{20}-1053139982 q^{22} \\
& -1543359636 q^{16}-718547767 q^{24}+40437585 q^{28}-625150182 q^{18}+10312920 q^{30} \\
& \left.-610724583 q^{12}-434987253 q^{8}-538477502 q^{10}+969300 q^{32}+97200 q^{34}\right) \\
& /\left(\left(2700 q^{14}+24945 q^{12}+73990 q^{10}+93072 q^{8}+46690 q^{6}+1267 q^{4}-5220 q^{2}-900\right) q^{25 / 2}\right) \\
& A_{5,3,1}=\frac{1}{65536}\left(108 q^{18}-5934 q^{16}+5105 q^{14}+46195 q^{12}-51879 q^{10}-35153 q^{8}\right. \\
& \left.+4283 q^{6}+7569 q^{4}+4239 q^{2}+891\right) /\left(\left(3+q^{2}\right) q^{21 / 2}\right) \\
& A_{5,3,5}=-\frac{1}{65536}\left(78144 q^{16}+364169 q^{14}-491094 q^{12}-1068351 q^{10}+1162018 q^{8}\right. \\
& \left.+332347 q^{6}-358650 q^{4}-114885 q^{2}-4050\right) /\left(q^{21 / 2}\left(4 q^{4}+23 q^{2}+15\right)\left(9 q^{2}+2\right)\right) \\
& b_{5,5,3}=-\frac{5}{196608}\left(342 q^{22}+1257 q^{20}+2085 q^{18}-31430 q^{16}-390368 q^{14}-350282 q^{12}\right. \\
& \left.+1479350 q^{10}+570136 q^{8}-1001838 q^{6}-419175 q^{4}+146205 q^{2}+12150\right) \\
& /\left(q^{10}\left(5 q^{8}+45 q^{6}+113 q^{4}+59 q^{2}-30\right)\right) \\
& b_{5,5,5}=\frac{5}{196608}\left(45 q^{16}-150 q^{14}-570 q^{12}+2618 q^{10}-3896 q^{8}-498 q^{6}+25866 q^{4}\right. \\
& \left.+8910 q^{2}+6075\right) /\left(\left(3 q^{4}+20 q^{2}+25\right) q^{12}\right) \\
& b_{5,1,1}=-\frac{1}{196608}\left(291600 q^{34}+2907900 q^{32}+32753160 q^{30}+149869395 q^{28}\right. \\
& -96175800 q^{26}-1388122677 q^{24}-1816814730 q^{22}-673012947 q^{20}-2204709010 q^{18} \\
& -4183229964 q^{16}-2019366082 q^{14}-127366501 q^{12}-1024292202 q^{10} \\
& \left.-1272297663 q^{8}-404176500 q^{6}+54476469 q^{4}+49965660 q^{2}+6779700\right) \\
& /\left(q^{12}\left(5 q^{2}+3\right)\left(5 q^{4}+5 q^{2}-2\right)\left(36 q^{4}+35 q^{2}+6\right)\left(3 q^{4}+20 q^{2}+25\right)\right) \\
& b_{5,3,5}=-\frac{3}{65536}\left(320 q^{16}+1737 q^{14}-4390 q^{12}-8591 q^{10}+157370 q^{8}+25203 q^{6}\right. \\
& \left.+112590 q^{4}+62451 q^{2}+2430\right) /\left(\left(36 q^{6}+215 q^{4}+181 q^{2}+30\right) q^{10}\right) \\
& b_{5,3,1}=-\frac{3}{65536}\left(20 q^{18}-1386 q^{16}+1159 q^{14}+17385 q^{12}-1473 q^{10}-24611 q^{8}\right. \\
& \left.+13245 q^{6}+5427 q^{4}+12393 q^{2}+2673\right) /\left(q^{10}\left(3+q^{2}\right)\right) \\
& b_{5,1,3}=\frac{1}{196608}\left(576 q^{16}+8184 q^{14}+17094 q^{12}+22645 q^{10}+2281 q^{8}+23658 q^{6}\right. \\
& \left.+7776 q^{4}+22761 q^{2}+2673\right) /\left(\left(4 q^{2}+3\right) q^{10}\right) \\
& b_{5,1,5}=\frac{1}{196608}\left(60 q^{12}+1728 q^{10}+781 q^{8}+16476 q^{6}-4158 q^{4}-4212 q^{2}-243\right)
\end{aligned}
$$




$$
\begin{aligned}
& /\left(\left(4 q^{2}+3\right) q^{10}\right) \\
& b_{5,3,3}=\frac{3}{65536}\left(216 q^{22}-570 q^{20}-18933 q^{18}-71519 q^{16}-100329 q^{14}-35063 q^{12}\right. \\
& \left.\quad+38725 q^{10}-123273 q^{8}-114291 q^{6}-157707 q^{4}-125388 q^{2}-43740\right) \\
& \quad /\left(q^{12}\left(35 q^{4}+84 q^{2}+45+4 q^{6}\right)\right) \\
& b_{5,5,1}=\frac{5}{196608}\left(108 q^{22}+648 q^{20}-3735 q^{18}+5853 q^{16}+84492 q^{14}+18828 q^{12}\right. \\
& \left.\quad-256546 q^{10}-31890 q^{8}+169632 q^{6}+96228 q^{4}+37665 q^{2}+3645\right) \\
& \quad /\left(\left(5 q^{4}+18 q^{2}+9\right) q^{10}\right)
\end{aligned}
$$

\section{B Specific List of Coefficients}

$g=9.81 \mathrm{~m} / \mathrm{s}^{2}, h=100 \mathrm{~m}, H=10 \mathrm{~m}, T=10 \mathrm{~s}$

$k=0.04068 \mathrm{~m}^{-1}, \varepsilon=0.2034, L=154.45 \mathrm{~m}$, Ursell Number $=0.23856$

\section{B.1 First Order}

$\mathrm{C}(1)=0.9997072 \mathrm{D}(1)=0$

$\mathrm{A}(1,1,1)=-1.000293 \mathrm{~b}(1,1,1)=1$

\section{B.2 Second Order}

$\mathrm{C}(2)=0 \mathrm{D}(2)=0.0001464075$

$\mathrm{A}(2,0,2)=0.25 \mathrm{~A}(2,2,2)=-0.0004396087$

$\mathrm{b}(2,2,0)=0.25 \mathrm{~b}(2,2,2)=0.2505862$

\section{B.3 Third Order}

$\mathrm{C}(3)=-0.1248168 \mathrm{D}(3)=0$

$\mathrm{A}(3,1,1)=0.4073222 \mathrm{~A}(3,1,3)=0.1566621$

$\mathrm{A}(3,3,1)=-7.331096 \mathrm{e}-05 \mathrm{~A}(3,3,3)=7.309631 \mathrm{e}-05$

$\mathrm{b}(3,1,1)=-0.3132697 \mathrm{~b}(3,1,3)=-0.06260989$

$\mathrm{b}(3,3,1)=0.2817996 \mathrm{~b}(3,3,3)=0.09408$

\section{B.4 Fourth Order}

$\mathrm{C}(4)=0 \mathrm{D}(4)=-0.0001192351$

$\mathrm{A}(4,0,2)=-0.1564883 \mathrm{~A}(4,0,4)=-0.0312155$

$\mathrm{A}(4,2,2)=0.1255568 \mathrm{~A}(4,2,4)=0.0269313$

$\mathrm{A}(4,4,2)=0.04177638 \mathrm{~A}(4,4,4)=-1.008631 \mathrm{e}-05$

$\mathrm{b}(4,2,0)=-0.1408818 \mathrm{~b}(4,2,2)=-0.3343606 \mathrm{~b}(4,2,4)=-0.05002256$

$\mathrm{b}(4,4,0)=0.1254033 \mathrm{~b}(4,4,2)=0.0837004 \mathrm{~b}(4,4,4)=0.04186642$ 


\section{B.5 Fifth Order}

$$
\begin{aligned}
& \mathrm{C}(5)=0.04277276 \mathrm{D}(5)=0 \\
& \mathrm{~A}(5,1,1)=-0.4809001 \mathrm{~A}(5,1,3)=-0.2658329 \mathrm{~A}(5,1,5)=-0.03957723 \\
& \mathrm{~A}(5,3,1)=-0.09390064 \mathrm{~A}(5,3,3)=-0.06788393 \mathrm{~A}(5,3,5)=0.003329216 \\
& \mathrm{~A}(5,5,1)=1.604243 \mathrm{e}-05 \mathrm{~A}(5,5,3)=5.088142 \mathrm{e}-05 \mathrm{~A}(5,5,5)=1.116865 \mathrm{e}-06 \\
& \mathrm{~b}(5,1,1)=0.3215159 \mathrm{~b}(5,1,3)=0.07839174 \mathrm{~b}(5,1,5)=0.007587707 \\
& \mathrm{~b}(5,3,1)=-0.2851435 \mathrm{~b}(5,3,3)=-0.2056731 \mathrm{~b}(5,3,5)=-0.03474901 \\
& \mathrm{~b}(5,5,1)=0.09991874 \mathrm{~b}(5,5,3)=-0.00231718 \mathrm{~b}(5,5,5)=0.0204687
\end{aligned}
$$

\section{References}

Fenton, J. D. (1985a). A fifth-order Stokes theory for steady waves. Journal of Waterway, Port, Coastal and Ocean Engineering, ASCE 111, 216-234. Errata, 113, p.438.

Fenton, J. D. (1985b). Wave forces on vertical walls. Journal of Waterway, Port, Coastal and Ocean Engineering, ASCE 111, 693-717.

Goda, Y. (1967). The fourth order approximation to the pressure of standing waves. Coastal Engineering in Japan 10, 1-11.

Goda, Y. and S. Kakizaki (1966). Study on finite amplitude standing waves and their pressures upon a vertical wall. Report of Port and Harbour Technical Research Institute, Yokosuka, Japan 5(10), 1-56. in Japanese.

Hsu, J. R. C., Y. Tsuchiya, and R. Silvester (1979). Third-order approximation to shortcrested waves. Journal of Fluid Mechanics 90, 179-196.

Isobe, M. and N. C. Kraus (1983). Derivation of a third-order Stokes wave theory. Technical Report YNU-HY-83-1, Department of Civil Engineering, Yokohama National University, Hydraulics Laboratory, Yokohama, Japan.

Mercer, G. N. and A. J. Roberts (1992). Standing waves in deep water: Their stability and extreme form. Physics of Fluids A 4, 259-269.

Penney, W. G. and A. T. Price (1952). Part II. Finite periodic stationary gravity waves in a perfect fluid. Philosophical Transactions, Royal Society, London (A) 244, 254-284.

Schwartz, L. W. and A. K. Whitney (1981). A semi-analytical solution for nonlinear standing waves in deep water. Journal of Fluid Mechanics 10\%, 147-171.

Tadjbakhsh, I. and J. B. Keller (1960). Standing surface waves of finite amplitude. Journal of Fluid Mechanics 8, 442-451.

Williams, J. M. (1985). Tables of Progressive Gravity Waves. Pitman, Boston. 